\title{
Erratum: Maximally symmetric two Higgs doublet model with natural standard model alignment
}

\author{
P.S. Bhupal Dev ${ }^{a}$ and Apostolos Pilaftsis ${ }^{a, b}$ \\ ${ }^{a}$ Consortium for Fundamental Physics, School of Physics and Astronomy, \\ University of Manchester, \\ Manchester, M13 9PL, United Kingdom \\ ${ }^{b}$ CERN, Department of Physics, Theory Division, \\ CH-1211 Geneva 23, Switzerland \\ E-mail: bhupal.dev@manchester.ac.uk, \\ apostolos.pilaftsis@manchester.ac.uk
}

ERRATUM TO: JHEP12(2014)024

ABSTRACT: Here we correct some typesetting errors in ref. [1]. These corrections have been implemented in the latest version of [1] on arXiv and the corrected equations have also been reproduced in ref. [2] for the reader's convenience. We clarify that all numerical results presented in ref. [1] remain unaffected by these typographic errors.

ARXiv EPRINT: 1408.3405 
- Eq. (2.10) in ref. [1] should be

$$
\begin{aligned}
-\mathcal{L}_{Y}^{q} & =\bar{Q}_{L}\left(h_{1}^{u} \widetilde{\Phi}_{1}+h_{2}^{u} \widetilde{\Phi}_{2}\right) u_{R}+\bar{Q}_{L}\left(h_{1}^{d} \Phi_{1}+h_{2}^{d} \Phi_{2}\right) d_{R} \\
& =\left(\bar{u}_{L}, \bar{d}_{L}\right)\left(\widetilde{\Phi}_{1}, \widetilde{\Phi}_{2}, \Phi_{1}, \Phi_{2}\right) \mathcal{H}\left(\begin{array}{c}
u_{R} \\
d_{R}
\end{array}\right),
\end{aligned}
$$

- In eq. (B.9), 3rd line, 2nd parenthesis should have an overall negative sign, i.e. $-\left(20 g_{3}^{2}+\frac{45}{8} g_{2}^{2}+\frac{85}{24} g^{\prime 2}\right) y_{t}^{2}$.

- In eq. (B.10), 6th line, the first term, i.e. $-20 \lambda_{1} \lambda_{3}^{2}$, which is redundant with the 5 th term, should be removed.

- In eq. (B.10), last line, the 3rd last term should be $+6 y_{t}^{2} y_{b}^{4}$.

- In eq. (B.11), 6th line, the first term, i.e. $-20 \lambda_{2} \lambda_{3}^{2}$, which is redundant with the 5 th term, should be removed.

- In eq. (B.11), 6th line, the 6 th term should be $3 \lambda_{4}$ instead of $3 \lambda_{3}$.

- In eq. (B.11), second last line, last term should be $+6 y_{b}^{2} y_{t}^{4}$.

- In eq. (B.12), 10th line, 4 th term should be $-5\left(3 g_{2}^{2}+5 g^{\prime 2}\right) \lambda_{3}$.

- In eq. (B.12), 11th line should have a leading factor of 2 and the superfluos parentheses before the braces should be removed, i.e. $-2\left\{2 \lambda_{3}^{2}+\lambda_{4}^{2}+4 \lambda_{1}\left(3 \lambda_{3}+\lambda_{4}\right)+\lambda_{5}^{2}+4 \lambda_{6}^{2}+8 \lambda_{6} \lambda_{7}\right\}\left(3 y_{b}^{2}+y_{\tau}^{2}\right)$.

- In eq. (B.13), last line, the brace for $-\frac{9}{2} \lambda_{4}$ term should end after $y_{\tau}^{4}$.

- In eq. (B.14), 7th line, last term, the coefficient of $\lambda_{5} \lambda_{6} \lambda_{7}$ should be 42 instead of 37 .

- In eq. (B.14), 9th line, the coefficient of second term should be $\frac{45}{4}$ instead of $\frac{45}{2}$.

- In eq. (B.15), 4th line, 2nd term should be $-\lambda_{2}^{2}$.

- In eq. (B.15), 8th line, 1st term, $\lambda_{4}$ should be removed. Similarly, the $\lambda_{4}$ after $14\left(\lambda_{1}+\lambda_{2}+2 \lambda_{3}\right)$ should be removed.

- In eq. (B.15), 2nd last line, inside 2nd parenthesis, the 1st term should be $24 \lambda_{1}$.

- In eq. (B.16), 4th line, the coefficient of the first group of terms should be $-36 \lambda_{3} \lambda_{6}$.

Open Access. This article is distributed under the terms of the Creative Commons Attribution License (CC-BY 4.0), which permits any use, distribution and reproduction in any medium, provided the original author(s) and source are credited. 


\section{References}

[1] P.S. Bhupal Dev and A. Pilaftsis, Maximally symmetric two Higgs doublet model with natural Standard Model alignment, JHEP 12 (2014) 024 [arXiv:1408.3405] [INSPIRE].

[2] P.S. Bhupal Dev and A. Pilaftsis, Looking for new naturally aligned Higgs doublets at the LHC, PoS (PLANCK 2015) 105 [arXiv: 1510.08790] [INSPIRE]. 